\title{
Harnessing multimodality to enhance quantification and reproducibility of in vivo molecular imaging data
}

\author{
We have combined the KoDAK In-Vivo Multispectral Imaging System FX with a new animal rotation \\ module. This module enables the precise co-registration of X-ray images with spectrally unmixed \\ fluorescence images of mice rotated through various craniocaudal angles. The X-ray images were \\ critical for reproducing and optimizing the spatial orientation of animals at angles that maximized the \\ luminescence intensity of the optical signal.
}

\begin{abstract}
We have previously described the importance of illumination calibration for enhancing quantitation and reproducibility for in vivo small animal molecular imaging (Wood, D., Feke, G., Vizard, D. \& Papineni, R. Refining epifluorescence imaging and analysis with automated multipleband flat-field correction. Nat. Methods 5; April 2008). Here we further the themes of quantitation and reproducibility by highlighting the value of multimodality and discuss the combination of X-ray and optical imaging modalities in the Kodak In-Vivo Multispectral Imaging System FX (http://www.carestreamhealth.com/).

In addition to yielding contextual anatomical information for localization of molecular imaging signals in animals, the X-ray modality may also be used to obtain information about the spatial orientation of the animals. Optimization and reproducibility of spatial orientation parameters such as the craniocaudal rotation angle are important because the detection of optical and radioisotopic signals in planar imaging is often sensitive to these experimental conditions. Thus, we have developed an animal rotation system to rapidly find the optimum animal angle with which to acquire optical signals for a given biological system. A key feature of animal rotation is that the system will accurately reproduce the position for the same animal during longitudinal studies requiring several time points.
\end{abstract}

Animal rotation for multiple-angle molecular imaging The animal rotation module that we developed operates on the existing platform of the Kodak In-Vivo Multispectral Imaging System FX. The apparatus includes a 29-mm diameter, 135-mm long torsional support that accommodates a recumbent mouse immobilized by anesthesia.

Gilbert D Feke, W Matthew Leevy, Sean Orton, Benjamin Geldhof, M Catherine Muenker, Manisha Patel, Rao Papineni, Douglas Wood, Douglas Vizard \& William McLaughlin

Carestream Molecular Imaging, 4 Science Park, New Haven, Connecticut 06511, USA Correspondence should be addressed to G.F. (gilbert.feke@carestreamhealth.com).

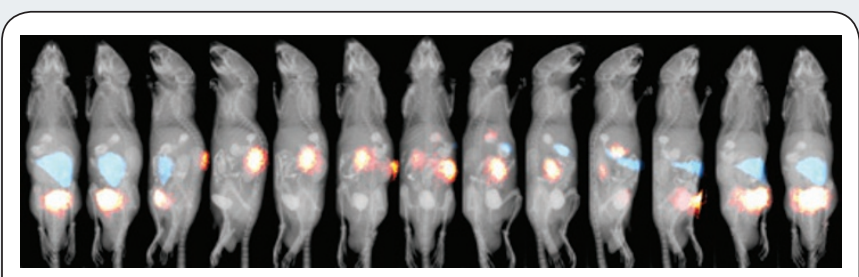

Figure 1 | Mouse administered X-ray contrast and fluorescence agents and rotated 360 degrees about craniocaudal axis. X-ray and spectrally unmixed fluorescence overlays are shown every 30 degrees. Fluorescence signal from the gastrointestinal tract (pseudocolored blue) and kidneys and urinary tract (pseudocolored red hot) fade in and out of view as the mouse rotates, owing to optical attenuation through the mouse body.

The animal may be incrementally rotated through 360 degrees through prone, supine and lateral orientations and those in between, by an automated protocol. The animal is not 'standing upright' but is instead recumbent to minimize positional stress. A set of multimodal images, including X-ray, multispectral fluorescence, luminescence and/ or radioisotopic images, may then be captured at each craniocaudal rotation angle. Each image set may be spectrally unmixed and overlaid on the X-ray image. Viewing a compilation of the images (Fig. 1), especially when displayed in sequence in a movie, provides a unique three-dimensional visualization of the molecular imaging signals and their anatomical context. Aside from the visual-interpretation advantages, the system enables the use of anatomical cues provided by the $X$-ray modality to detect and define the spatial orientation of the animal at the various rotational angles.

\section{Determining spatial orientation from X-ray images}

Straightforward comparison of X-ray images provides a rich methodology to assess animal spatial orientation. For example, dimensional analysis of bone arrangement or calculation of image differences may be used to calculate rotation angle. Beyond this, we can use systematic algorithms to quantify spatial orientation. For example, we obtained 

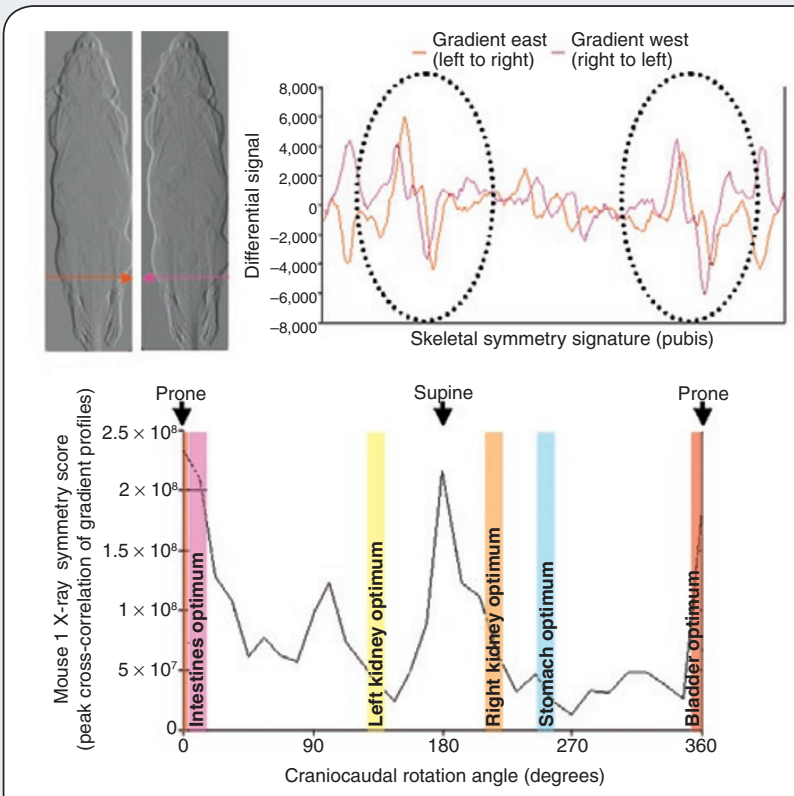

Figure $\mathbf{2}$ | Examples of gradient-filtered X-ray images (top left) and the corresponding line profiles through the pubis bone (top right) are shown. The dashed ovals highlight the skeletal symmetry signature. A series of such line profiles through an angular scan can be analyzed for bilateral symmetry to systematically determine craniocaudal rotation angle, as shown in the bottom graph

line profiles through the mouse pubis bone in gradient-filtered X-ray images (Fig. 2). Analysis of the bilateral symmetry of the rotational series of such line profiles yields a doubly peaked function (shown unwrapped) indicating the prone and supine orientations (maximal bilateral symmetry). Any arbitrary craniocaudal rotation angle may be interpolated between the prone and supine orientations.

\section{Optimizing and reproducing spatial orientation}

To demonstrate the value of multimodality, we administered KODAK $\mathrm{X}$-SIGHT imaging agents in combination with $\mathrm{X}$-ray contrast agents to three different mice. One mouse was fed a meal of creamy peanut butter mixed with barium sulfate (1:1 ratio by weight; Orton, $\mathrm{S}$. et al. Facile imaging of murine bone and soft tissue using the Kodak Digital X-Ray Specimen system. Biocompare Online, http://www. biocompare.com/technicalarticle/1658/Facile-Imaging-Of-MurineBone-And-Soft-Tissue-Using-The-Kodak-Digital-X-Ray-SpecimenSystem-from-Carestream-Molecular-Imaging-(formerly-KodakMolecular-Imaging-Systems).html) and Kodak X-Sight 761 nanospheres to delineate the gastrointestinal tract (stomach and intestine) with X-ray and fluorescent contrast agents, respectively. To contrast the urinary system, a second mouse received a tail vein injection consisting of iodixanol (Visipaque) (320 milligrams of iodine per milliliter) X-ray contrast agent mixed with Kodak X-Sight 670 Large Stokes Shift (LSS) dye. A third mouse received both administrations. The spectrally unmixed and overlaid images are shown in Figure $\mathbf{1 .}$

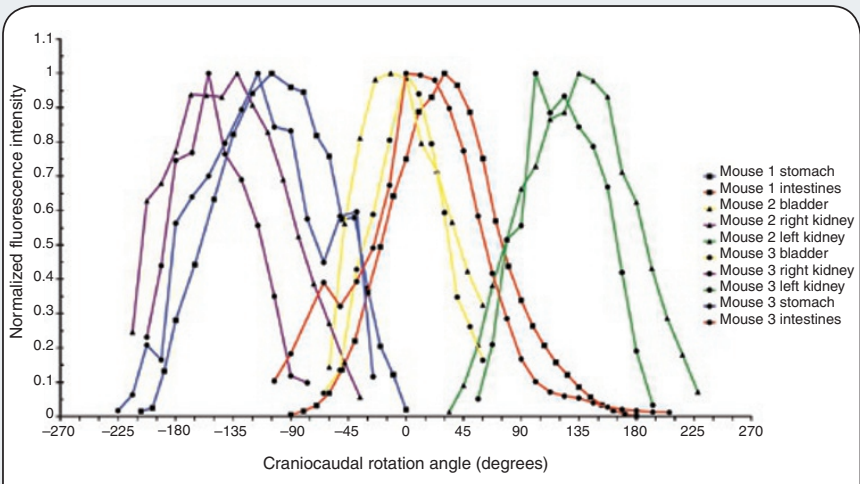

Figure $\mathbf{3}$ | Fluorescence signals depend on craniocaudal rotation angle. Optimal rotation angles are reproducible among multiple mice.

In each case, the X-ray contrast agents were included to demonstrate the anatomical origin of the fluorescent signals in these soft tissues, which have poor innate contrast by X-ray. During imaging of each mouse, we observed that each organ had an optimal viewing angle at which maximum signal was attained (Fig. 3). These optimal viewing angles are consistent among the mice (by virtue of the fact that the internal organs are generally arranged in the same way). These optimal viewing angles are represented as bands in Figure 2. Sweeping through a range of views ensured that the maximum optical signal was captured. Furthermore, the use of X-ray images provided cues for the spatial orientation, thus enabling convenient replication of spatial orientation for the same mouse at a later time point.

\section{Conclusions}

Carestream Molecular Imaging has developed and commercialized powerful multimodal in vivo imaging systems and products that greatly enhance the localization, quantitation and reproducibility of molecular signals in live animals. These Kodak-branded systems are now used by top academic, biotechnology and pharmaceutical research institutes worldwide. The flexibility of the system allows the combination and co-registration of multiple wavelengths, multiple imaging modalities and now multiple angles with which to perform imaging studies.

Visipaque is a trademark of GE Healthcare. Carestream and X-Sight are trademarks of Carestream Health. The Kodak trademark is used under license from Kodak. Carestream Molecular Imaging is a division of Carestream Health, Inc. Although the Kodak In-Vivo Multispectral Imaging System FX can be used for in vivo and in vitro molecular imaging of materials, researchers should be aware that the methods of preparing and viewing the materials for molecular imaging may be subject to various patent rights. All images were captured using Kodak Molecular Imaging Products.

This article was submitted to Nature Methods by a commercial organization and has not been peer reviewed. Nature Methods takes no responsibility for the accuracy or otherwise of the information provided. 